\title{
Evaluation of Causality Measures Based on Non-Uniform Embedding Schemes with Application to the Cardiovascular System
}

\author{
Petroula Laiou, Ralph G. Andrzejak and Dimitris Kugiumtzis*
}

\begin{abstract}
In the analysis of physiological time series it is important to investigate the inter-dependence structure among the observed variables of the system. For this, a number of measures of the so-called Granger causality have been developed, and among them information measures have gained much attention. However, information measures have to deal with the estimation of probability distributions of highdimensional vector variables, typically formed through uniform delay embedding of the time series. Here, the focus is on measures derived after non-uniform embedding. Particularly, two schemes are considered, one based on conditional mutual information and the other based on conditional entropy. We evaluate the two measures on simulated nonlinear coupled dynamical systems and apply them to signals of heart rate variability, respiration, and oxygen concentration in the blood.
\end{abstract}

\section{INTRODUCTION}

The inter-dependence structure of systems underlying multivariate time series has been traditionally investigated by Granger causality. Granger causality, first applied in econometrics, states that the variable $X$ Granger causes the variable $Y$, if the driving variable $X$ improves the prediction of the response variable $Y$ when $X$ is included in the autoregressive model of $Y$. The concept of causality has been extended in recent years, and apart from different established measures (e.g. [1], [2], [3]), information measures of Granger causality became popular, mainly because they are modelfree and nonlinear. However, these measures rely on vector variables $\mathbf{x}_{t}$ and $\mathbf{y}_{t}$ at each time $t$ formed from the time series $\left\{x_{t}, y_{t}\right\}_{t=1}^{n}$ of $X$ and $Y$ through time delay embedding, i.e. $\mathbf{x}_{t}=\left[x_{t}, x_{t-\tau}, \ldots, x_{t-(m-1) \tau}\right]$ for embedding dimension $m$ and a fixed time delay $\tau$, and the same for $Y$. The most prominent information causality measure transfer entropy (TE) is defined as $\mathrm{TE}_{X \rightarrow Y}=I\left(y_{t+1} ; \mathbf{x}_{t} \mid \mathbf{y}_{t}\right)$, where $I(Y ; X \mid Z)$ is the conditional mutual information (CMI), i.e. the mutual information (MI) of variables $Y$ and $X$ given $Z$. The estimation of $I(Y ; X \mid Z)$ requires the estimation of entropies and thus probability distributions of vector variables, the largest being the one concatenating $X, Y$, $Z$. For the setting of TE this requires the estimation of probability distributions of the variable of dimension $2 m+1$, and thus TE estimation can fail depending on $m$ and $n$.

As an alternative to uniform embedding of each variable separately, a mixed non-uniform embedding of $X$ and $Y$ with

R.G. Andrzejak acknowledges grant FIS-2010-18204 of the Spanish Ministry of Education and Science

P. Laiou and R.G. Andrzejak are with the Department of Information and Communication Technologies, Universitat Pompeu Fabra, Barcelona, Spain.

D. Kugiumtzis is with the Department of Electrical and Computer Engineering, Aristotle University of Thessaloniki, Greece (*corresponding author e-mail: dkugiu@auth.gr). the purpose of explaining the future of $Y$ was developed in [4] using CMI to select the components in the mixed embedding vector from $X$ and $Y$. The causality measure was formed as a normalized version of TE defined on mixed embedding, called mutual information from mixed embedding (MIME), and later extended to partial MIME (PMIME) for multi-variate time series [5]. In [6], a similar approach was presented using instead the conditional entropy (CE). This approach was applied to investigate cardiovascular and cardiorespiratory interactions [6], [7]. Here, we compare the two approaches in assessing inter-dependencies among heart rate variability, respiration, and blood oxygen concentration. We confine the causality analysis to pairs of variables using the corresponding bivariate time series, so we do not consider the conditioning on other variables.

\section{Methods}

Although the causality measures MIME in [4] and FNP (after the author's last names) in [6] are based on nonuniform embedding and information measures, they have some structural differences. MIME uses CMI to build a mixed embedding vector $\mathbf{w}_{t}$ from delays of both $X$ and $Y$ and then restricts the vector variable for $X$, denoted $\mathbf{w}_{t}^{x}$, to include the delay components in $\mathbf{w}_{t}$ stemming from $X$, and the same for $Y$, denoted $\mathbf{w}_{t}^{y}$. FNP builds $\mathbf{w}_{t}$ progressively as MIME but the lag components of $\mathbf{w}_{t}$ are selected on the basis of CE rather than CMI. Furthermore, $\mathbf{w}_{t}^{y}$ is not a subset of $\mathbf{w}_{t}$, but it is derived repeating the same selection process restricted on delays of $Y$ alone.

The measure MIME is defined as the TE but on the restricted embedding for $X$ and $Y$ and normalized by the total MI

$$
R_{X \rightarrow Y}=\frac{I\left(y_{t+1} ; \mathbf{w}_{t}^{x} \mid \mathbf{w}_{t}^{y}\right)}{I\left(y_{t+1} ; \mathbf{w}_{t}\right)},
$$

It takes values between zero, when $\mathbf{w}_{t}$ does not contain delay components of $X$ ( $X$ does not contribute in explaining $y_{t+1}$ and thus there is no causal effect), and one when $\mathbf{w}_{t}$ does not contain delay components of $Y$ ( $X$ fully determines $Y$ ).

The measure FNP is defined as

$$
C_{X \rightarrow Y}=1-\frac{H^{c}\left(y_{t+1} \mid \mathbf{w}_{t}\right)}{H^{c}\left(y_{t+1} \mid \mathbf{w}_{t}^{y}\right)}
$$

where $H^{c}$ denotes the corrected conditional entropy (for details see [6]). If there is no causality $X \rightarrow Y, \mathbf{w}_{t}$ is expected to contain only delay components of $Y$ and then $\mathbf{w}_{t}=\mathbf{w}_{t}^{y}$, giving $C_{X \rightarrow Y}=0$. However, the upper bound of $C_{X \rightarrow Y}$ is always smaller than one, because $H^{c}\left(y_{t+1} \mid \mathbf{w}_{t}\right)$ is always nonzero. Another main difference of MIME and FNP is in the estimation of the entropies. MIME uses the $k$ nearest neighbors estimate ( $\mathrm{kNN}$ ) while FNP uses equidistant 
binning. The $\mathrm{kNN}$ estimate is found appropriate to deal with high dimensional vector variables, while equidistant binning, like any other discretization approach, is more data demanding.

\section{Simulations}

In [8] a study on MIME and FNP was conducted using some of the discrete and continuous time dynamical systems used in [4], [6]. Also, the two measures were evaluated on different time series lengths. For length $n=1000$ they had very good sensitivity (identifying causality when present) and specificity (not identifying spurious causality), while for $n=$ 100 they still retained some ability. This is shown in Fig. 1 for the coupled Henon map, where the true coupling is $X \rightarrow$ $Y$. Significance test, with significance level $\alpha=0.05$ and
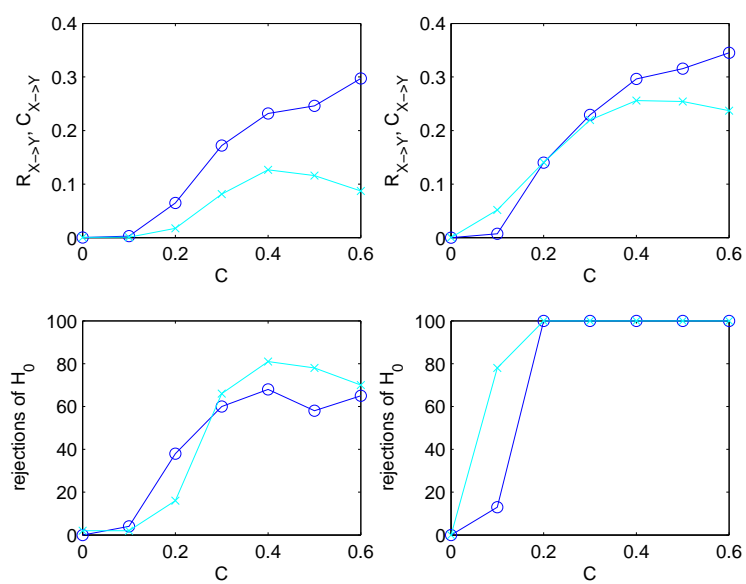

Fig. 1. Mean $R_{X \rightarrow Y}$ and $C_{X \rightarrow Y}$ and rejections of the $H_{0}$ for 100 realizations of the uni-directionally coupled Henon map [4] versus coupling strength $C$ (०: MIME, $\times$ : FNP). The largest lag for both measures is 10 . For MIME the number of neighbors is 1 for $n=100$ and 10 for $n=1000$. The termination threshold is $A=0.95$. For FNP the number of bins is 6 . First column: $n=100$, Second column: $n=1000$. For $n=1000,10 \%$ Gaussian white noise was added.

null hypothesis, $\mathrm{H}_{0}: C=0$ for $R_{X \rightarrow Y}$ and $C_{X \rightarrow Y}$ using time-shifted surrogates, confirmed that for $n=1000$ both measures give no rejection when the coupling strength $C$ is zero, and they reject $\mathrm{H}_{0}$ in all realizations when $C \geq 0.2$. When $n=100$, the two measures behave quite the same. Similar results, were obtained from other systems, and it is noted that $C_{X \rightarrow Y}$ could at cases be negative, probably due to negative bias of the binning estimate of conditional entropy.

\section{CAUSAlity IN THE CARDIOVASCULAR SYSTEM}

The inter-dependence of all pairs of heart variability $(X)$, respiration $(Y)$ and blood oxygen concentration $(Z)$ were assessed with MIME and FNP on data taken from the Santa Fe Competition [9]. Two stationary segments of $X, Y$ and $Z$ were selected and normalized from the whole recording, A: samples 2350-3550 showing signs of apnea, B: samples 12500-13700 showing rather normal activity. The parameters for MIME and FNP and the significance test are as in the simulation study at $n=1000$. The results are shown in Table I, where the star denotes the rejection of the $H_{0}$ at $\alpha=0.05$.
TABLE I RESULTS FOR THE SEGMENTS A AND B OF HEART RATE (X), BREATH RATE (Y), AND BLOOD OXYGEN CONCENTRATION (Z).

\begin{tabular}{|c|cc|cc|}
\hline & \multicolumn{2}{|c|}{$\mathrm{A}$} & \multicolumn{2}{c|}{$\mathrm{B}$} \\
& MIME & FNP & MIME & FNP \\
\hline$X \rightarrow Y$ & 0.11 & 0.01 & 0 & -0.01 \\
$Y \rightarrow X$ & 0 & -0.01 & $0.21 \star$ & $0.10 \star$ \\
\hline$X \rightarrow Z$ & 0 & -0.00 & 0.05 & 0.00 \\
$Z \rightarrow X$ & 0 & 0.06 & 0 & 0.03 \\
\hline$Y \rightarrow Z$ & 0 & 0 & 0 & 0.02 \\
$Z \rightarrow Y$ & $0.12 \star$ & 0.02 & 0 & $0.03 \star$ \\
\hline
\end{tabular}

Both causality measures detect driving effect from the breath rate to the heart rate, a phenomenon which is called respiratory sinus arrhythmia [10]. Although MIME takes positive value for the inverse direction, this value is not significant according to the significance test. Also, both of them do not detect causality between the heart rate and the blood oxygen concentration. For the last pair, they find causality from the blood oxygen concentration to the respiration, but for different segments.

\section{CONCLUSIONS}

We evaluated two measures, MIME and FNP, for the estimation of Granger causality based on non-uniform embedding. MIME uses conditional mutual information and FNP conditional entropy. The evaluation was performed on simulations, and it was found that the two measures have better sensitivity and specificity when the length of the data is large. For small time series they retain some accuracy. The differences in the performance of MIME and FNP resulted in different coupling detection on the three time series of heart rate, breath rate and oxygen concentration in the blood. Both measures agreed that the respiration affects the heart, and the blood oxygen concentration affects the breath rate.

\section{REFERENCES}

[1] T. Kreuz, F. Mormann, R.G. Andrzejak, A. Kraskov, K. Lehnertz, and P. Grassberger, "Measuring synchronization in coupled model systems: A comparison of different approaches," Physica D, vol. 225, no. 1, pp. 29-42, 2007.

[2] D. Chicharro and R.G. Andrzejak, "Reliable detection of directional couplings using rank statistics," Phys. Rev. E, vol. 80, p. 026217, 2009.

[3] A. Papana, C. Kyrtsou, D. Kugiumtzis, and C. Diks, "Simulation study of direct causality measures in multivariate time series," Entropy, vol. 15 , no. 7, pp. 2635-2661, 2013.

[4] I. Vlachos and D. Kugiumtzis, "Non-uniform state space reconstruction and coupling detection," Phys. Rev. E, vol. 82, p. 016207, 2010.

[5] D. Kugiumtzis, "Direct-coupling information measure from nonuniform embedding," Phys. Rev. E, vol. 87, p. 062918, 2013.

[6] L. Faes, G. Nollo, and A. Porta, "Information-based detection of nonlinear Granger causality in multivariate processes via a nonuniform embedding technique," Phys. Rev. E, vol. 83, p. 051112, 2011.

[7] - "Compensated transfer entropy as a tool for reliably estimating information transfer in physiological time series," Entropy, vol. 15, no. 1, pp. 198-219, 2013.

[8] P. Laiou, "Comparison of two granger causality indices that combine mixed embedding and information measures (in Greek)," Master thesis, Department of Physics, Aristotle University of Thessaloniki, 2013.

[9] D. R. Rigney, A. L. Goldberger, W. Ocasio, Y. Ichimaru, G. B. Moody, and R. Mark, Time Series Prediction: Forecasting the Future and Understanding the Past. Addison-Wesley, 1993, ch. 3, p. 105130.

[10] R. W. deBoer, J. M. Karemaker, and J. Strackee, "Hemodynamic fluctuations and baroreflex sensitivity in humans: a beat-to-beat model," American Journal of Physiology - Heart and Circulatory Physiology. vol. 253, pp. H680-H689, 1987 